\title{
EVALUACIÓN DE LA ADIPONECTINA Y SU CORRELACIÓN CON LA ÍNTIMA MEDIA CAROTIDEA EN MUJERES PERI Y POST MENOPÁUSICAS. HOSPITAL DE ENGATIVÁ, BOGOTÁ
}

\begin{abstract}
EVALUATION ADIPONECTIN AND ITS CORRELATION WITH CAROTID INTIMA MEDIA IN WOMEN PERI-AND POSTMENOPAUSAL. ENGATIVA HOSPITAL, BOGOTA
\end{abstract}

\author{
William Onatra Herrera ${ }^{1}$, Jacinto Sánchez Angarita ${ }^{2}$, Mario Parra Pineda $^{3}$, Jairo Amaya Guio ${ }^{4}$, Gladys Laverde ${ }^{5}$
}

\begin{abstract}
${ }^{1}$ MD, M.Sc., Profesor Titular Ginecología y Obstetricia. Universidad de Ciencias Aplicadas y Ambientales U.D.C.A, calle 222 No. 55 - 30, Campus Norte. Universidad Nacional de Colombia, e-mail: wonatra@udca.edu.co; ${ }^{2}$ MD, Ph.D., Profesor Titular Ginecología y Obstetricia. Universidad Nacional de Colombia. Coordinador Programa de Incontinencia Urinaria y Menopausia. Hospital de Engativá, Bogotá, carrera 30 No. 45-03, Bloque 471, e-mail: jsancheza@unal.edu.co; ${ }^{3}$ MD, M.Sc., Profesor Asociado. Universidad Nacional de Colombia. Ecografista. Hospital Engativá, carrera 30 No. 45-03, Bloque 471, e-mail: moparra@unal.edu.co; ${ }^{4}$ MD, Ph.D., Profesor Titular Ginecología y Obstetricia. Universidad Nacional de Colombia. Coordinador Programa de Colposcopia, carrera 30 No. 45-03, Bloque 471, e-mail: jamayaguio@unal.edu.co; ${ }^{5}$ Directora Laboratorio de Investigaciones Hormonales, Bogotá, carrera 7C No. 121-34, e-mail: gerencia@lablih.net
\end{abstract}

Rev. U.D.C.A Act. \& Div. Cient. 19(2): 257-266, Julio-Diciembre, 2016

\section{RESUMEN}

El riesgo cardiovascular (RCV), se incrementa en las mujeres después de la menopausia. La adiponectina, se postula como un biomarcador de riesgo cardiovascular. La presente investigación buscó conocer si los niveles de adiponectina y la evaluación de la Intima Media Carotidea (IMT) se modifican durante el climaterio. Se evaluó el riesgo cardiovascular a diez años, en la escala de Framingham (FRS). Se tomaron 80 pacientes, entre 45 y 65 años, de la consulta de Climaterio en el Hospital de Engativá, Bogotá, entre el 15 de enero y 15 de diciembre de 2015. Las pacientes, se dividieron en dos grupos: 46 perimenopáusicas y 34 posmenopáusicas, todas las participantes firmaron el consentimiento informado. En formulario precodificado, se consignaron las variables sociodemográficas, examen físico, valores química sanguínea y resultados de ecografía doppler de carótida. La edad media entre los dos grupos fue: 50,95 años y 60,59 años. Se evidenció diferencia significativa en la presión arterial media. No hubo diferencia estadística en el perímetro abdominal ni en el Índice de Masa Corporal (IMC). La adiponectina presentó diferencia significativa intra grupos. No hubo diferencia en el perfil lipídico, el riesgo cardiovascular aumentó significativamente entre las mujeres pre y postmenopáusicas. La evaluación de la Íntima Media Carotidea (IMT) evidenció diferencias significativas en la placa anterior y posterior, tanto de carótida derecha como de carótida izquierda. El índice de pulsatilidad (IP) fue significativo en la carótida derecha y no en la izquierda. Se postula que la adiponectina es un marcador de riesgo cardiovascular y la IMT útil en la evaluación de arterioesclerosis.

Palabras clave: Climaterio, adiponectina, perfil lipídico, ecografía carotidea, Íntima Media Carotidea (IMT).

\section{SUMMARY}

The cardiovascular risk (CVR) increases in women after menopause. Adiponectin is postulated as a biomarker of cardiovascular risk. This study sought to determine whether adiponectin levels and assessment of Carotid Intima Media (IMT) are modified during the climacteric. cardiovascular risk ten years in the Framingham (FRS) was evaluated. 80 patients between 45 and 65 years of consulting Climaterio at the Hospital of Engativá, Bogota, between January 15 and December 15, 2015. The patients were divided into two groups, 46 perimenopausal and 34 postmenopausal, all participants signed informed consent. In pre-encoded form sociodemographic variables, physical examination, blood chemistry values and results of carotid doppler ultrasound were recorded. The average age between the two groups was: 50.95 years and 60.59 years. Significant difference was evidenced in mean arterial pressure. There was no statistical difference in waist circumference or the Body Mass Index (BMI). Adiponectin showed a significant difference intra groups. There was no difference in lipid profile, cardiovascular 
risk increased significantly between pre- and postmenopausal women. Evaluation of Carotid Intima Media showed significant differences in the anterior and posterior plates both right and left carotid carotid. The pulsatility index (PI) was significant in the right carotid artery and not in the left. We hypothesize that adiponectin is a marker of cardiovascular risk and IMT useful in assessing atherosclerosis.

Key words: Menopause, adiponectin, lipid profile, carotid ultra-sonography, Carotid Intima Media (IMT).

\section{INTRODUCCIÓN}

El tejido adiposo no es solo un depósito de almacenamiento de energía sino que tiene una serie de funciones complejas, en todos los órganos del cuerpo humano. Su comunicación con otros sistemas biológicos está acoplada a la expresión de varios mediadores, llamados adipoquinas o adipocitoquinas. Existen una veintena de adipocitoquinas y factores inflamatorios, dentro de las cuales, se pueden destacar: la adiponectina, leptina, resistina, interleuquina 6 (IL-6), factor de necrosis tumoral alfa (TNF-@) y el inhibidor del activador de plasminogeno-1 (PAI-1) (Antoniades et al. 2009). La adiponectina es una proteína parecida a la del colágeno, producida por el adipocito durante su diferenciación Diferentes estudios posteriores demostraron el papel benéfico sobre los vasos arteriales, considerándose como una molécula antiaterogénica y regulador en la homeostasis de los hidratos de carbono (Yamauchi et al. 2002).

Los diferentes eventos metabólicos están mediados por la adhesión de la adiponectina unida a dos tipos de receptores: receptor uno ADIPOR1, se encuentra en el músculo esquelético, cuya activación aumenta la captación de la glucosa y la beta oxidación de los ácidos grasos; el receptor ADIPOR2, presente en el hígado, donde disminuye la gluconeogénesis. El gen de la adiponectina está localizado en el cromosoma $3 q 27$, correspondiente a un locus de susceptibilidad de enfermedades metabólicas, demostrándose la asociación de polimorfismos genéticos con la obesidad, diabetes mellitus tipo II y eventos cardiovasculares (Harvest, 2009).

La adiponectina presenta, experimental y clínicamente, una serie de funciones, que incluyen: la modificación en la expresión de moléculas de adhesión endotelial y la inhibición en la proliferación de músculo liso en los vasos arteriales; regula la homeostasis de los hidratos de carbono, vía resistencia a la insulina (Yamauchi et al. 2002); disminución de lipoproteínas, con efectos sobre la enfermedad cardiovascular (ECV), demostrándose un efecto protector. Por otra parte, en los casos de hipoadiponectinemia, se encontró una relación asociada a la dislipidemia, síndrome metabólico (Yang \& Chuang, 2006), hipertensión, enfermedad coronaria y vascular periférica (Kampoli et al. 2009).
La enfermedad cardiovascular (ECV) es la primera causa de morbimortalidad después de la menopausia. Esta observación fue confirmada en el estudio de Framingham, en los cuales, las mujeres posmenopáusicas tenían dos a seis veces más ECV que las mujeres premenopáusicas, con aumento de los eventos coronarios, después de los 70 años, superando a los hombres. Se ha postulado una serie de factores que contribuyen a esta patología, destacándose, en primer lugar, el efecto protector de los estrógenos, a nivel endotelial, que disminuyen al llegar la menopausia (Collins et al. 2007).

El término arteriosclerosis, se describe como un aumento del grosor de las arterias, a medida que aumenta la edad. Este fenómeno es debido a la aparición de placas de ateroma que produce una vasoconstricción arterial, que lleva a hipertensión arterial y, por otro lado, disminuye el flujo sanguíneo, favoreciendo la trombosis. Se han postulado tres factores principales en la formación de placa: Externos: estilo de vida, nutrición, estrés, alcohol, medioambiente, cigarrillo, drogadicción. Genéticos: enzima convertidora de angiotesina (ECA), apoliproteina E, E-selectina, matriz de metaloproteinasa 3 (MPP-3), matriz gla proteína, receptor a de estrógenos, ectonucleotido pirofosfatasa-fosfodiesterasa, epoxido hidroxilasa y osteoprotegerina. Endocrino-metabólicos: estrógeno, hormona paratiroidea, adiponectina, vitamina D, colesterol total (CT), lipoproteínas de alta densidad (HDL), lipoproteínas de baja densidad (LDL) y triglicéridos (TG), homocisteina y radicales libres (Weber \& Noels, 2011).

Otro de los factores atribuibles en la ECV es el impacto que tienen los esteroides sexuales sobre las lipoproteínas de CT, con una disminución de las lipoproteínas HDL, aumento de las LDL y TG (Gupta \& Smith, 2014). Estos cambios favorecen la formación de placa ateromatosa, a nivel endotelial.

La evaluación de riesgo cardiovascular ha tenido diferentes parámetros en su evaluación, que incluye: escala clásica y por categorías de Framingham, sociedades europeas, británicas, Nueva Zelanda y Sheffield. La variables tenidas en cuenta para riesgo coronario en la forma clásica del Framingham, comprende: edad, sexo, presión arterial sistólica y diastólica, CT, HDL, tabaquismo y diabetes; con estos parámetros, se puede calcular el riesgo coronario a 5 y 10 años, que abarca, angina inestable, infarto de miocardio (IAM) y muerte coronaria (Coema, 2011).

La deficiencia de estrógenos, se encuentra asociada a un aumento de peso, del tejido adiposo visceral (Gambacciani et al. 1999), niveles de homocisteina, aumento de la resistencia a la insulina y tensión arterial (Reckelhotff, 2004).

El ultrasonido de carótida ha sido de gran ayuda en la evaluación de la arterioesclerosis sistémica. El aumento en el grosor de la Íntima Media Carotidea (IMT) es un buen pre- 
dictor de un futuro infarto miocárdico (Iglesia del Sol et al. 2002). La baja ecogenicidad y la superficie irregular, se encuentran asociadas a un evento futuro cardiovascular. Esta técnica presenta una sensibilidad $71 \%$ y una especificidad $63,6 \%$, para detección de estenosis coronaria, en pacientes diabéticos (Fujihara et al. 2013). Ha sido interés de los autores conocer la influencia de la adiponectina sobre el riesgo cardiovascular y IMT en mujeres pre y postmenopáusicas, en una población de bajos ingreso, en el Hospital de Engativá, Bogotá.

\section{MATERIALES Y MÉTODOS}

El estudio fue de corte transversal y para el cálculo de la muestra, se tuvo en cuenta la proyección del número de mujeres entre 45 y 65 años, en el área de Bogotá, ajustada al área de influencia del Hospital de Engativá.

Sujetos participantes: Se seleccionaron 80 mujeres, quienes asistieron a consulta externa de ginecología, con edades entre 45 y 65 años, en el hospital de Engativá, entre el 15 de enero y 15 de diciembre del 2015. Criterios de inclusión: Mujeres entre 45 y 65 años, diagnóstico de premenopausia y postmenopausia, índice de masa corporal (IMC) entre 19 y 39. Criterios de exclusión: dislipidemia familiar, diabetes mellitus I o II, diabetes MODYS, alcoholismo, drogadicción, cardiopatías congénitas, insuficiencia cardiaca congestiva, enfermedad coronaria, vascular periférica y cerebrovascular. Análisis clínico: edad, procedencia, grupo étnico, antecedentes de tabaquismo, dislipidemia, médico, quirúrgico, IMC. Para el cálculo de riesgo cardiovascular a diez años, se utilizó la escala de Framingham (Framingham Scale, 2010). Análisis paraclínico: CT, HDL, LDL, TG, Glicemia $(\mathrm{G})$, adiponectina sérica, proteína $\mathrm{C}$ reactiva ultrasensible (PCRu) (Yang \& Chuang, 2006). Como muestras, se tomaron $5 \mathrm{~mL}$ de sangre venosa, entre las siete y nueve de la mañana, ayuno mínimo de cuatro horas y no haber realizado ejercicio 24 horas antes de la prueba. Para las mediciones del perfil lipídico y la glicemia, se utilizaron kits comerciales Cobas C11 Roche, Indianápolis IN, USA. Valores de normalidad: Glicemia $110 \mathrm{mg} / \mathrm{dL}$ o 3,9nmol/L), Colesterol Total $<200 \mathrm{mg} / \mathrm{dL}$ o $5,17 \mathrm{nmol} / \mathrm{L}, \mathrm{HDL}>65 \mathrm{mg} / \mathrm{dL}$ o $>1,55 \mathrm{nmol} / \mathrm{L}$, LDL $<150 \mathrm{mg} / \mathrm{dL}$ o $2,59 \mathrm{nmol} / \mathrm{L}$ y Triglicéridos $<150 \mathrm{mg} / \mathrm{dL}$ o 0,45nmol/L. Para la Adiponectina, 5-30ug/mL y PCR ultrasensible, $1,0 \mathrm{mg} / \mathrm{dL}$, se utilizó un test de ELISA comercial (Linco Research,Missouri (USA), en un lector de placas de ELISA, Synergy 2 (Bioteck,Vermont VT,USA) (Yamauchi et al. 2002).

Valores de la adiponectina: Mujeres entre 50 y 59 años, de $2-23 \mu \mathrm{g} / \mathrm{mL}$, mayores de 60 años, entre $11,2-24,1 \mu \mathrm{g} / \mathrm{mL}$. Análisis ecográfico de la carótida común: se utilizó un ecógrafo Siemens Sonoline G-50, con un transductor lineal 5-10 $\mathrm{MHz}$ y se evaluaron en las arterias carótidas comunes, en vecindad a su bifurcación: los diámetros externo e interno, el grosor combinado de la íntima y la media (complejo I-M) de las paredes anterior y posterior y la resistencia al flujo, a través de la determinación del Índice de Pulsatilidad (IP). El grosor del complejo I-M, se consideró anormal un valor mayor de $0,8 \mathrm{~mm}$, asociado a un mayor riesgo de un proceso arterosclerótico (Mac Ananey et al. 2014).

Análisis estadístico: Se realizó de acuerdo al software SPSS versión 17. Los datos iniciales fueron sometidos al test de normalidad de Shapiro-Wilk. Las variables categóricas se representaron en medias y desviación estándar. La diferencia entre las dos poblaciones premenopausia vs postmenopausia, se calculó con la T-Student, para muestras independientes con sus respectivos intervalos de confianza, al $95 \%$. El nivel de significancia, se definió como $\mathrm{p}<0.05$ (Ruiz \& Morillo, 2004).

Componente ético: Se tuvo en cuenta la resolución 8430 de 1993 del Ministerio de Salud, artículo 15, correspondiente al consentimiento informado y sus componentes, el cual, fue firmado por todas las pacientes participantes en el estudio. De acuerdo a la misma resolución, este estudio se puede catalogar como de riesgo mínimo, dado que solo se realizaron procedimientos no invasivos, salvo la extracción de sangre, para la toma de adiponectina y PCR ultrasensible y los parámetros bioquímicos. Este estudio fue presentado y autorizado por los comités de investigaciones de ética de la U.D.C.A y del Hospital de Engativá. El estudio, se rige por los principios de la declaración de Helsinki 1994 y del código de Núremberg para investigación médica, garantizando un trato respetuoso a los pacientes y evitando cualquier situación, que pueda comprometer su integridad física o mental.

\section{RESULTADOS Y DISCUSIÓN}

La mujer, después de la menopausia, presenta una serie de eventos, como: oleadas de calor, insomnio, irritabilidad, artralgias, aumento de peso, hipertensión, colesterol total, lipoproteínas de baja densidad (LDL) o aterogénicas y triglicéridos, disminución de las lipoproteínas de alta densidad (HDL) o protectoras, calcificación de las arterias, disminución del flujo endotelial, que generan aumento del riesgo cardiovascular. La adiponectina es una adipocitoquina secretada por los adipocitos en el tejido adiposo, con efecto en la regulación de la sensibilidad de la insulina, homeostasis de la glucosa, perfil lipídico, hipertensión, nivel vascular endotelial. Los bajos niveles, se encuentran asociados a la progresión de la diabetes y aumento del riesgo cardiovascular (Zhang et al. 2012).

El climaterio, entendido como un periodo de transición entre el final de la vida reproductiva, 45-55 años y la vejez, mayores de 65 años, ha tenido diferentes clasificaciones, 
como premenopausia, menores de 50 años; menopausia, promedio 50 más o menos 5 años; postmenopausia, mayores de 50 años y perimenopausia, entre los 45 y 55 años (Soules et al. 2001). En el 2001, en un Consenso aceptado por la Asociación Norteamericana de Menopausia (NAMS), de acuerdo al último periodo menstrual, consideró: menos de uno a dos años, transición menopaúsica y más de uno a dos años, postmenopausia (Soules et al. 2001). La edad de la menopausia, en un estudio multicéntrico latinoamericano, en 17.150 mujeres, entre 40 y 59 años, evidenció que la edad promedio fue de 49,4+/-5,5 años siendo la más baja en Asunción (Paraguay), con 43,8 años y la más alta, en Cartagena (Colombia), de 53 años (Castelo-Branco et al. 2006). Para efecto del presente estudio, las pacientes se consideraron perimenopaúsicas, entre los 45 y 54 años y postmenopáusicas, entre los 55 y 65 años. La edad promedio de la menopausia en el primer grupo fue de 50,95+/-10,2 años y el segundo, de 60,59+/-12,5 años, que está de acuerdo con un estudio japonés (Kotani et al. 2011), en un grupo similar de 252 pacientes climatéricas y describe que el promedio de edad de las mujeres premenopaúsicas era de 44,7+/-4,9 años y de las postmenopáusicas, de 64,6+/-4,4 años, con diferencia significativa $(p<0,001)$.

La hipertensión es un problema de salud pública extendido en el mundo o asociado a riesgo cardiovascular. El aumento de la tensión arterial, se presenta después de la menopausia, atribuido a la disminución de los estrógenos, aumento de lípidos, placa arteriosclerótica y a un incremento en el Índice de Masa Corporal y tejido adiposo. La adiponectina es una adipocitoquina, con propiedades antiinflamatorias, antiateromatosas asociadas a alteraciones cardiometabólicas, incluyendo la hipertensión (Yiannikouris et al. 2010). La tensión arterial media fue para el grupo de las premenopaúsicas, de 89,17+/-9,24 y en las postmenopáusicas, de $94,86+/-11,57$ con diferencia significativa; coincide, con el estudio de Kotani et al. (2011), quienes también encontraron diferencias significativas entre las mujeres pre y postmenopáusicas, de $87,6+/-11,0 \mathrm{~mm} . \mathrm{Hg}$ y 100,7+13,7mm.Hg, con una $\mathrm{p}<0,001$ (Tabla 1).

Tabla 1. Descripción de las diferentes variables del estudio.

\begin{tabular}{|c|c|c|c|}
\hline Variables & $\begin{array}{l}\text { Perimenopáusicas } \\
45-54 \text { años } \\
n=46\end{array}$ & $\begin{array}{l}\text { Postmenopáusicas } \\
55-65 \text { años } \\
n=34\end{array}$ & $\mathrm{P}<0001$ \\
\hline Edad años & 50,95 & 60,59 & \\
\hline T.A. Media $\mathrm{mmHg}$ & $89,17+/-9,24$ & $94,86+/-11,57$ & $0,017^{*}$ \\
\hline IMC & $27,47+/-4,45$ & $26,60+/-4,81$ & 0,452 \\
\hline P. Abdominal cm & $91,96+/-11,22$ & $90,50+/-12,59$ & 0,587 \\
\hline P. Cadera. cm & $101,46+/-8,25$ & $99,09+7,94$ & 0,201 \\
\hline Cintura/Cadera & $0,91+0,08$ & $0,91+/-0,09$ & 0,764 \\
\hline \multicolumn{4}{|c|}{ ADIPONECTINA, PCR Y PERFIL LIPÍDICO } \\
\hline Adiponectina ug/mL & $11,09+/-6,01$ & $13,49+/-5,56$ & $0,074 *$ \\
\hline PCR & $5,33+/-5,52$ & $3,44+/-5,26$ & 0,118 \\
\hline Glicemia & $93,89+/-11,51$ & $101,97+/-34,17$ & 0,140 \\
\hline Colesterol $\mathrm{T}$ & $219,35+/-46,42$ & $228,44+/-43,87$ & 0,378 \\
\hline HDL & $57,69+/-19,43$ & $54,47+/-14,85$ & 0,422 \\
\hline LDL & $134,39+/-43,08$ & $136,64+/-37,04$ & 0,807 \\
\hline Triglicéridos & $144,39+/-50,72$ & $172,91+/-100,63$ & 0,101 \\
\hline $\mathrm{CT} / \mathrm{HDL}$ & $4,06+/-1,18$ & $4,46+/-1,35$ & 0,156 \\
\hline E.Framingham \% & $3,07+/-1,57$ & $5,56+/-3,35$ & 0,001 \\
\hline \multicolumn{4}{|c|}{ ECOGRAFÍA Y DOPPLER CARÓTIDA } \\
\hline CD-Placa Anterior mm & $0,51+/-0,22$ & $0,74+/-0,25$ & $0,001 *$ \\
\hline CD-Placa Posterior mm & $0,68+/-0,19$ & $0,86+/-0,26$ & 0,001 \\
\hline CI-Placa Anterior mm & $0,55+/-0,22$ & $0,78+/-0,41$ & $0,002 *$ \\
\hline CD-Placa posterior & $0,68+/-0,19$ & $0,97+/-0,39$ & 0,001 \\
\hline CD-IP & $1,37+/-0,24$ & $1,51+/-0,28$ & $0,022 *$ \\
\hline CI-IP & $1,42+/-0,30$ & $1,50+/-0,23$ & 0,187 \\
\hline
\end{tabular}

* Diferencia significativa 
El aumento de peso, se asocia al Síndrome Metabólico (SM), hipertensión, arteriosclerosis y enfermedad cardiovascular. El tejido adiposo, fuera de ser un depósito de energía, produce una serie de adipocitoquinas, mencionadas al inicio de este estudio, que se encuentran comprometidas en la patogénesis de las enfermedades del tejido adiposo, resistencia a la insulina, inflamación crónica, disfunción cardiovascular (Ouchi et al. 2011). El presente estudio evidencia que no hay diferencia estadística en el IMC, entre las mujeres perimenopausicas, 27,47+/-4,45 y postmenopausicas, 26,60+/$4,81(\mathrm{p}<0,452)$, corroborado por el estudio de Kotani et al. (2011), que halla $23,4+/-4,3$ y $24,0+/-3,5$ ( $p<0,207)$, en paciente pre y posmenopáusicas, respectivamente (Tabla 1 ).

En la medición del perímetro abdominal, como medida indirecta y proporcional al perfil lípido y al tejido adiposo abdominal, no hay diferencia entre las pacientes perimenopausicas y postmenopáusicas $(91,96+/-11,22 \mathrm{~cm}$ y $90,50+/-12,59 \mathrm{~cm}$, $\mathrm{p}<0,587$, respectivamente); estas medidas, se encuentran por encima del corte establecido para la mujer latinoamericana, que es de $80 \mathrm{~cm}$ (Blümel et al. 2012). Tampoco, se evidenciaron diferencias en el perímetro de cadera y en la relación cintura/cadera (Tabla 1). Estos datos coinciden con el estudio latinoamericano, donde Colombia presenta una de las tasas más altas de Síndrome Metabólico, con un 45,6\% (Royer et al. 2007), cuando la media es 35,1\%.
Los estudios experimentales y clínicos evidencian que la adiponectina es una hormona multifuncional con efectos en diferentes órganos de la economía, siendo los más estudiados la disminución de peso y tejido graso a nivel cerebral, glucosa hepática, ácidos grasos y triglicéridos por supresión de genes gluconeogenéticos. A nivel cardiaco, ejerce un efecto cardioprotector sobre las fibras del miocardio, con aumento de la angiogénesis, en casos de infarto. En el área reproductiva, se han hallado receptores de adiponectina, en la hipófisis hipotálamo, útero y ovario. Es conocido el efecto sobre patologías ginecológicas, como miomatosis uterina, endometriosis y cáncer endometrial. Disminuye la producción de la hormona liberadora de gonadotropinas $(\mathrm{GnRH})$, con efectos sobre la hormona luteinizante (LH) y la hormona estimulante de los folículos (FSH). Clínicamente, no afecta el ciclo menstrual, pero interviene en la proliferación, esteroideogénesis y maduración del oocito.

El presente estudio confirmó que los niveles de adiponectina se elevan durante la pos $\mu$ menopausia, con diferencia significativa $11,09+/-6,01 \mu \mathrm{g} / \mathrm{mL}$ y $13,49+/-5,56 \mu \mathrm{g} / \mathrm{mL}$, respectivamente $(\mathrm{p}<0.074)$ (Tabla 1) (Gráfica 1), confirmando lo expuesto por Angelidis et al. (2013).

Datos también reportados por Kotani et al. (2011), quienes describen los niveles de adiponectina en la premenopausia,

\section{Comparacion de Niveles de Adiponectina. Pre y Post menopausia}

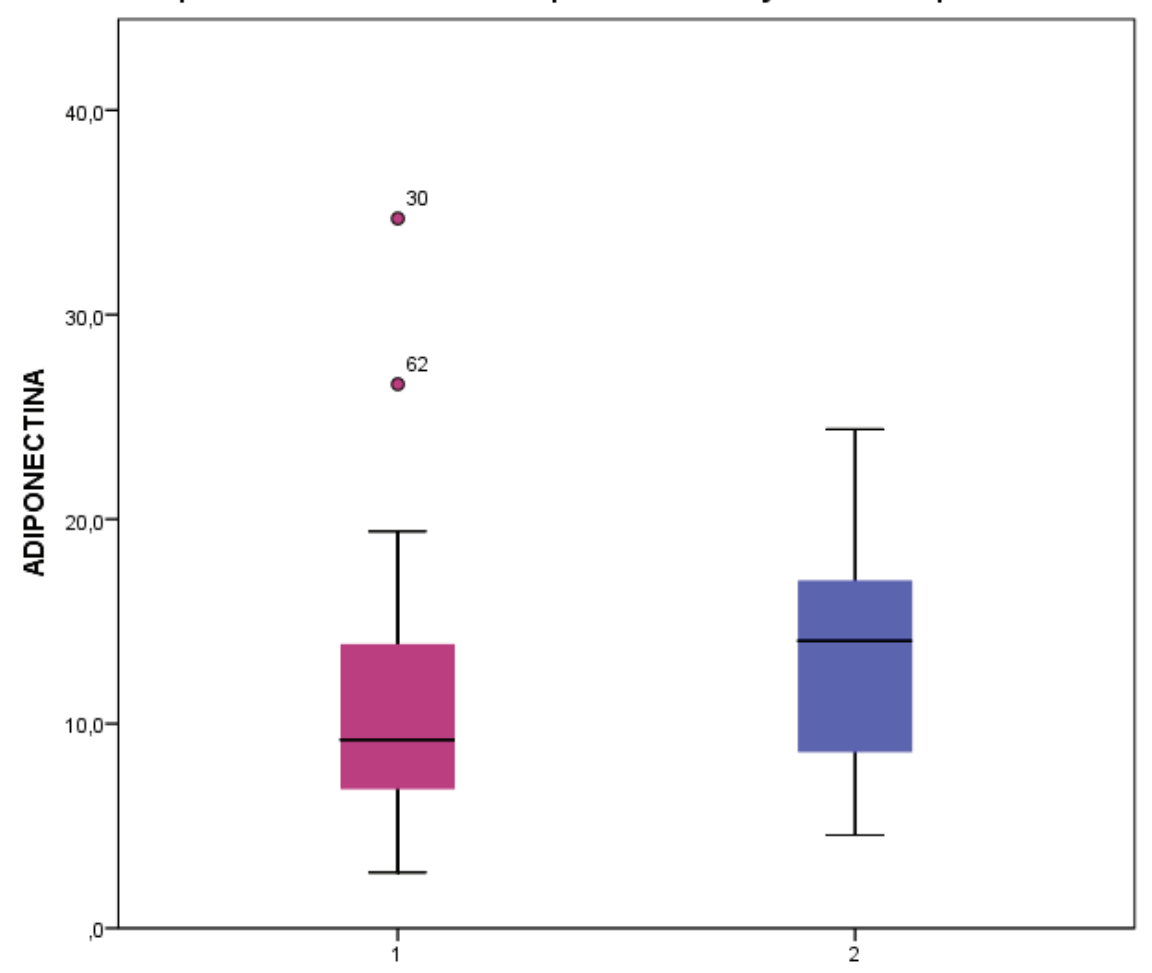

Gráfica 1. Niveles de adiponectina, por grupo de edad, 1 pre, 2 post-menopáusica. 
de $8,8+/-5,6 \mu \mathrm{g} / \mathrm{mL}$ y en la postmenopausia, $9,9+/-6,5 \mu \mathrm{g} /$ $\mathrm{mL}$, con diferencia significativa $(\mathrm{p}<0,015)$. Wu et al. (2015), en 119 mujeres durante la premenopausia, registraron niveles de $8+/-6 \mu \mathrm{g} / \mathrm{L}$ y en 62 postmenopáusicas, niveles de $13+/-5 \mu \mathrm{g} / \mathrm{L}$, con diferencia significativa $(\mathrm{p}<0,05)$. En estas condiciones, la adiponectina se perfila como un biomarcador durante el climaterio, con modificaciones en los casos de hipertensión, sobrepeso, obesidad y arteriosclerosis.

El síndrome metabólico es una entidad caracterizada por aumento de tensión arterial, glicemia, triglicéridos (TG) y disminución de las HDL, entidades que, en conjunto, proporciona la formación de placa arteriosclerótica y riesgo cardiovascular. Al desaparecer, los valores de estrógenos y la progesterona, después de la menopausia se modifican, suscitando el daño cardiometabólico (Onatra et al. 2006).

El presente estudio evaluó la glicemia, el perfil lipídico y el índice CT/HDL, evidenciando que el Colesterol total se encuentra por encima de los valores de referencia, $200 \mathrm{mg} / \mathrm{dl}$ o 5,17nmol/L; una tendencia al aumento, que también se presenta con las LDL y los triglicéridos $(0,5-2 \mathrm{nmol} / \mathrm{L})$. Las HDL, por el contrario, tienden a disminuir, sin ser significativas, en los casos de mujeres postmenopáusicas (Tabla1). Estos datos coinciden con el estudio de Kotani et al. (2011), donde no hallaron diferencias estadísticas entre las mujeres premenopáusicas y postmenopáusicas, en el colesterol total, $5,08+/-0,4 \mathrm{nmol} / \mathrm{L}$ vs $5,06+/-0,57 \mathrm{nmol} / \mathrm{L}, \quad(p<0,534)$, ni en las HDL, $1,67+/-0,29 \mathrm{nmol} / \mathrm{L}$ vs $1,61+0,40 \mathrm{nmol} / \mathrm{L}$, $(\mathrm{p}<0,163)$. En cambio, evidenciaron diferencias significativas en la glicemia, $4,92+/-0,42 \mathrm{nmol} / \mathrm{L}$ vs $5,10+0,46 \mathrm{nmol} / \mathrm{L}$, $(\mathrm{p}<0,001)$ y los triglicéridos, $0,84+0,62 \mathrm{nmol} / \mathrm{L}$ vs $1,03+0,8 \mathrm{nmol} / \mathrm{L},(\mathrm{p}<0,001)$. Es de anotar que adiponectina, se aprecia elevada en este grupo etario en las mujeres postmenopáusicas, que explicaría, parcialmente, las escasas modificaciones en el perfil lipídico, que coincide con lo reportado por Funahashi \& Matsuzawa (2014).

La evaluación del riesgo cardiovascular en la Escala de Framingham ha sido parámetro universal aceptado en la evaluación del riesgo cardiovascular, a pesar de existir otras escalas aplicadas en otros países, como la europea, británica y Nueva Zelandia. El factor de riesgo para enfermedad coronaria a 10 años, se clasifica en bajo riesgo $<10 \%$, riesgo medio 10 $20 \%$ y alto riesgo $>20 \%$. El presente estudio evidenció que el riesgo cardiovascular se elevó significantemente entre las mujeres pre y postmenopáusicas, $3,07+/-1,57$ vs $5,56+/$ 3,35 , $(p<0,001)$ y que éstas se encontraban en riesgo bajo, $5 \%$ y $4 \%$, respectivamente, de acuerdo con los hallazgos de otras investigaciones (Cagnacci et al. 2015) (Tabla 1).

Diferentes estudios experimentales y clínicos evidencian que la adiponectina tiene acciones antiaterogénicas, por inhibición de la placa ateromatosa. Estos efectos "in vivo" demuestran que la adiponectina inhibe: la adhesión de monocitos, inducida por el factores de necrosis tumoral alfa (TNFa); la transformación de macrófagos, en células espumosas; la migración y la proliferación de células en músculo liso y activa la síntesis de óxido nítrico, a nivel endotelial. Clínicamente, se ha evidenciado que la adiponectina es un marcador para identificar etapas tempranas de arteriosclerosis; sus niveles están disminuidos en pacientes con hipertensión arterial, diabetes mellitus tipo II, enfermedad arterial coronaria, fumadoras, existiendo controversia en pacientes con sobrepeso y obesidad. La enfermedad cardiovascular, se incrementa después de la menopausia por diferentes factores: Internos: neuroendocrinos, ante la disminución de los esteroides gonadales; disfunción del perfil lipídico con modificaciones, a nivel endotelial y formación de placa ateromatosa; Externos: como los nutricionales, estilos de vida y el sedentarismo, contribuyen a la aceleración de esta placa, a medida que aumenta la edad.

En la evaluación de placa arterioesclerótica, se han utilizado diferentes métodos, como la evaluación de la Íntima Media Carotidea (IMT) por ultrasonido, Tomografía Axial computarizada (TAC) y Emisión de positrones (PET) en arterias coronarias. En el presente estudio, se utilizó la IMT, para la evaluación de placa en ambas carótidas, de mujeres pre- menopáusicas y postmenopáusicas, evidenciándose que existen diferencias significativas entre las placas anterior, $0,51+/-0,22 \mathrm{~mm}$ vs $0,74+/-0,25 \mathrm{~mm}$, ( $p<0,001)$, posterior de la carótida derecha, $68+/-0,19 \mathrm{~mm} . v \mathrm{~s} 0,86+/-0,26 \mathrm{~mm}$, $(\mathrm{p}<0,001)$ y en las placas anterior, $0,55+/-0,22 \mathrm{~mm}$ vs $0,78+/-0,41 \mathrm{~mm},(\mathrm{p}<0,002)$ y posterior, $0,68+/-0,19 \mathrm{~mm}$ vs $0,97+0,39, \mathrm{~mm},(\mathrm{p}<0,001)$, de carótida izquierda. El índice de pulsatilidad (IP) fue significativo en la carótida derecha $1,37+/-0,24$ vs $1,51+/-0,28$, ( $p<0,022$ ), no en la carótida izquierda $(p<0,187)$, posiblemente, porque anatómicamente existe un mayor flujo en la carótida izquierda con menor posibilidad de formación de placa (Tabla 1) (Gráficas 2 y 3 ) (Foto 1).

El estudio realizado por Johnson et al. (2010), en 72 mujeres peri menopáusicas y 131 postmenopáusicas, utilizando el IMT, demuestran el aumento progresivo de placa, a nivel carotideo. Estos hallazgos permiten confirmar, en nuestro medio, que la mujer, durante su periodo postmenopáusico, aumenta la placa arterioesclerótica, que llevará a mediano y largo plazo, desarrollar enfermedad cardiovascular y es conocida como la primera causa de morbimortalidad en mujeres mayores, por ello, se hace necesario recomendar medidas preventivas, como estilos de vida saludable, dieta baja en azúcares y grasas, ejercicio y el uso de medicamentos, para evitar la progresión de placa arterioriosclerótica.

De la presente investigación, se concluye que la adiponectina se perfila como una proteína marcadora útil en la evalua- 


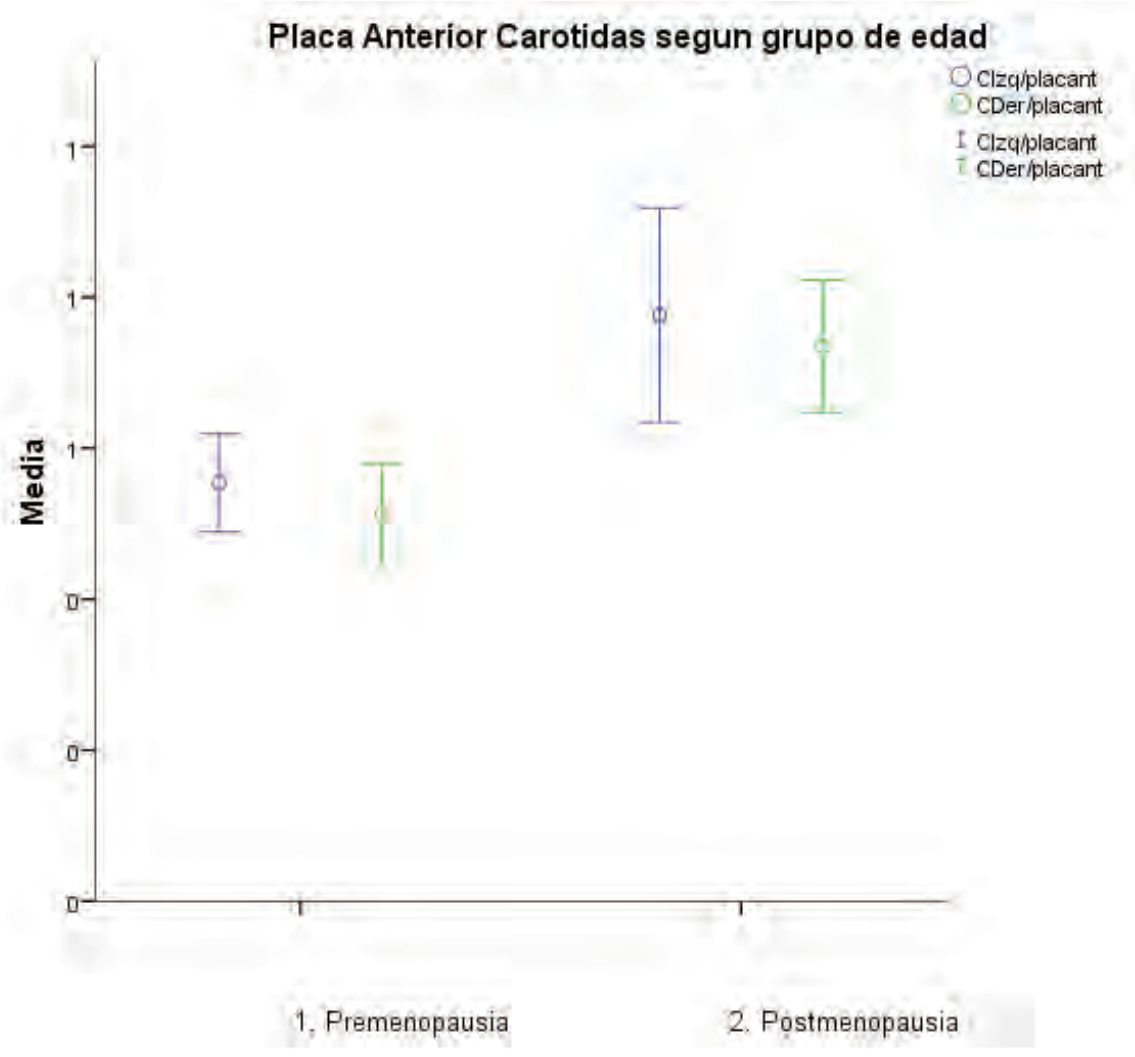

Gráfica 2: Formación de placa en carótida anterior, por grupo de edad.

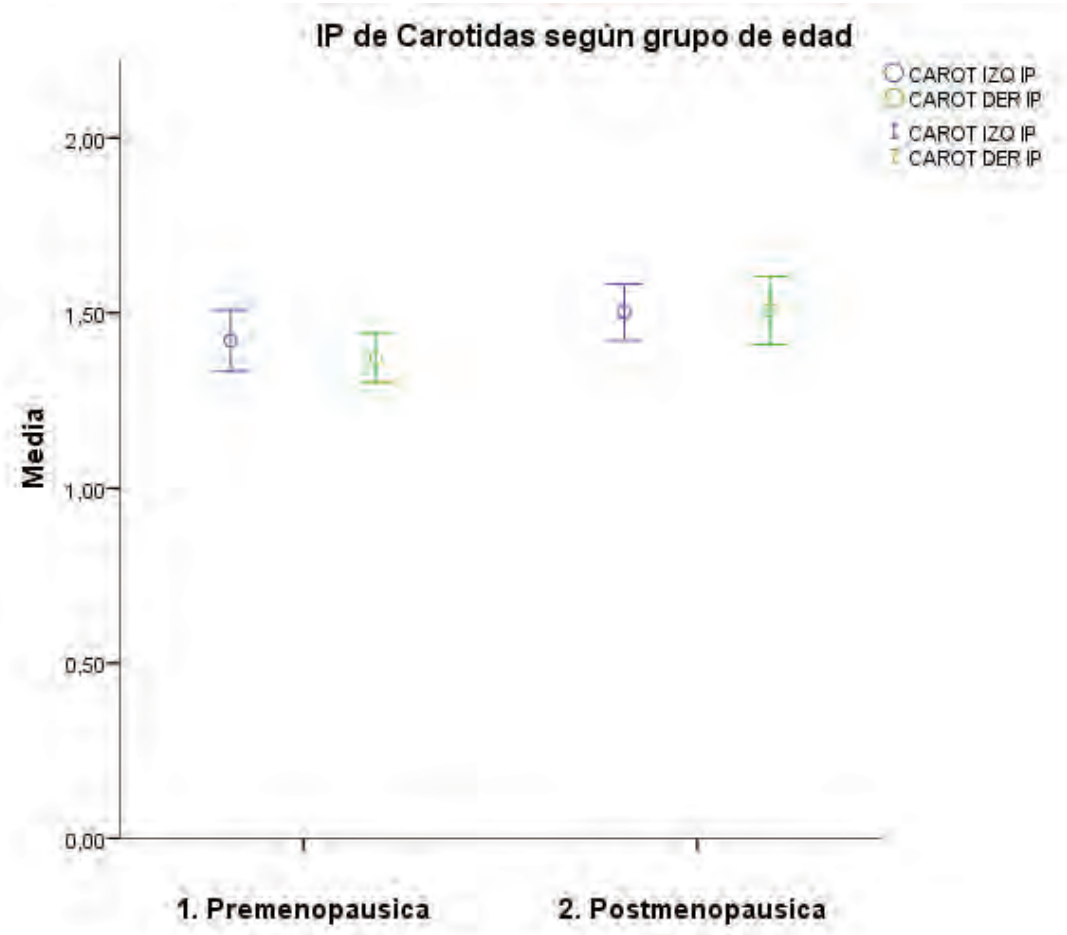

Gráfica 3: Índice de pulsatilidad en Carótidas Izquierda y Derecha, por grupo de edad. 


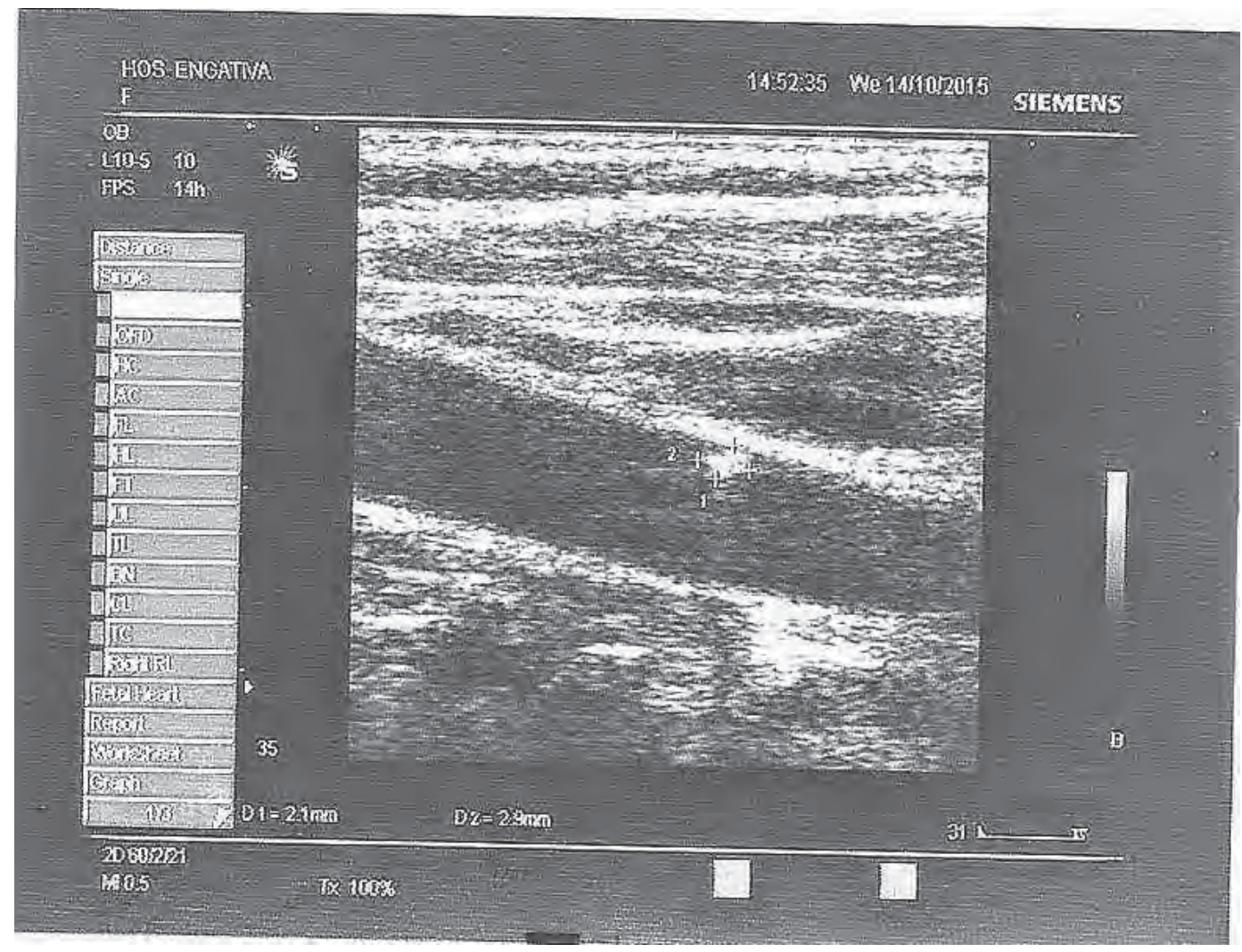

Foto 1: Ultrasonido de carótida con formación de placa.

ción de la mujer, durante el climaterio, donde presenta elevación de sus niveles, evidenciando el inicio de un deterioro progresivo metabólico endotelial; por otra parte, podría ser útil como marcador de respuesta terapéutica. La escala de Framingham, para riesgo cardiovascular, sigue siendo útil y el estudio mostró que después de la menopausia el riesgo es bajo, pero aumenta con la edad. La evaluación de la Íntima Media Carotidea, por ecografía doppler, es un procedimiento que permite evaluar indirectamente el estado coronario y el estudio permitió detectar la formación de placa, en forma significativa, en Carótida Derecha, con disminución del índice de pulsatilidad, confirmando el inicio de la arterioesclerosis e hipertensión arterial. Será de interés ampliar esta muestra por edad y por estratos, con el fin de tomar medidas preventivas, para evitar la formación de placa.

Agradecimientos: Los autores agradecen a las directivas y a la Vicerrectoría de Investigaciones de la Universidad de Ciencias Aplicadas y Ambientales U.D.C.A, directivas y Comité de Investigaciones del Hospital de Engativá, por el apoyo logístico y financiero, para llevar a cabo esta investigación. Al laboratorio de investigaciones hormonales, al personal de enfermería y laboratorio clínico del Hospital de Engativá. Conflictos de intereses: El manuscrito fue preparado y revisado con la participación de todos los autores, quienes declaramos que no existe conflicto de intereses que ponga en riesgo la validez de los resultados presentados.

\section{BIBLIOGRAFÍA}

1. ANGELIDIS, G.; DAFOPOULOS, K.; MESSINI, C.I.; VALOTASSIOU, V.; TSIKOURAS, P.; VRACHMIS, N.; PSIMADAS, D.; GEORGOULIAS, P.; MESSINIS, I.E. 2013. The emerging roles of adiponectin in female reproductive system-associated disorders and pregnancy. Reproductive Sciences. 20(8):872-881.

2. ANTONIADES, C.; ANTONOPOULOS, A.S.; TOUSOULIS, D.; STEFANADIS, C. 2009. Adiponectin: from obesity to cardiovascular disease. Obes. Rev. 10(3):269-279.

3. BLÜMEL, J.E.; LEGORRETA, D.; CHEDRAUI, P.; AYALA, F.; BENCOSME, A.; DANCKERS, L.; LANGE, D.; ESPINOZA, M.T.; GOMEZ, G.; GRANDIA, E.; IZAGUIRRE, H.; MANRIQUEZ, V.; MARTINO, M.; NAVARRO, D.; OJEDA, E.; ONATRA, W.; POZZO, E.; PRADA, M.; ROYER, M.; SAAVEDRA, J.M.; SA- 
YEGH, F.; TSEROTAS, K.; VALLEJO, M.S.; ZUÑIGA, C.; COLLABORATIVE GROUP FOR RESEARCH OF THE CLIMACTERIC IN LATIN AMERICA (REDLINC). 2012. Optimal waist circumference cutoff value for defining the metabolic syndrome in postmenopausal Latin American women. Menopause. 19 (4):433-437.

4. CAGNACCI, A.; PALMA, F.; ROMANI, C.; XHOLLI, A.; BELLAFRONTE, M.; DI CARLO, C. 2015. Are climacteric complaints associated with factors of cardiovascular disease in peri-menopausal women? Gynecol. Endocrinol. 31(5):359-362.

5. CASTELO-BRANCO, C.; BLÜMEL, J.E.; CHEDRAUI, P.; CALLE, A.; BOCANERA, R.; DEPIANO, E.; FIGUEROA-CASAS, P.; GONZALEZ, C.; MARTINO, M.; ROYER, M.; ZUÑIGA, C.; DULON, A.; ESPINOZA, M.T.; FUTCHNER, C.; MOSTAJO, D.; SOTO, E.; ALBERNAZ, M.A.; ARAVENA, H.; BUSQUETS, M.; CAMPODONICO, I.; GERMAIN, A.; ALBA, A.; BARON, G.; GOMEZ, G.; MONTERROSA, A.; ONATRA, W.; BROUTIN, G.; MANZANO, B.; GABRIELA, A.; HIDALGO, L.; LEON, P.; ORBEA, M.; SANCHEZ, H.; VALLEJO, S.; VALLECILLO, G.; HERNANDEZBUENO, J.; MOTTA, E.; ANDRADE, R.; TSEROTAS, K.; GONZALEZ, M.C.; BENITEZ, Z.; CALLE, E.; DANCKERS, L.; DEL CASTILLO, A.; IZAGUIRRE, H.; OJEDA, E.; ROJAS, J.; BENCOSME, A.; LIMA, S.; MOTTA, E. 2006. Age at menopause in Latin America. Menopause. 13(4):706-712.

6. COEMA, A.A. 2011. Las tablas de riesgo cardiovascular: una revisión crítica. MEDIFAM. 11(2):122-139.

7. COLLINS, P.; ROSANO, G.; CASEY, C.; DALY, C.; GAMBACCIANI, M.; HADJI, P.; KAAJA, R.; MIKKOLA, T.; PALACIOS, S.; PRESTON, R.; SIMON, T.; STEVENSON, J.; STRAMBA-BADIALE, M. 2007. Management of cardiovascular risk in the peri-menopausal woman: a consensus statement of European cardiologists and gynecologists. Eur. Heart J. 28(11):20282040.

8. FRAMINGHAM SCALE. 2010. Disponible desde Internet en: http://www.mdcalc.com/framingham-coronaryheart-disease-risk-score/ (con acceso 22/02/2016).

9. FUJIHARA, K.; SUZUKI, H.; SATO, A.; KODAMA, S.; HEIANZA, Y.; SAITO, K.; IWASAKI, H.; KOBAYASHI, K.; YATOH, S.; TAKAHASHI, A.; YAMADA, N.; SONE, H.; SHIMANO, H. 2013. Carotid artery plaque and LDL to HDL colesterol ratio predict arteriosclerotic status in coronary arteries in asyntomatic patients with type 2 diabetes Mellitus. J. Atheroscler. Thromb. 20(5):452-464.

10. FUNAHASHI, T.; MATSUZAWA, Y. 2014. Adiponectin and the cardiometabolic syndrome: An epidemiological perspective. Best Pract \& Res. Clin. Endocrinol. \& Metab. 28(1):93-106.

11. GAMBACCIANI, M.; CIAPONI, M.; GAPPAGLI, B.; BENUSSI, C.; DE SIMONE, L.; GENAZZANI, A. 1999. Climateric modifications in body weight and fat tissue distribution. Climateric. 2(1):37-44.

12. GUPTA, A.; SMITH, D.A. 2014. The 2013 American college of cardiology american hearth association. Guidelines on treating blood cholesterol and assessing cardiovascular risk. Endocrin. Metab. Clin. N. Am. 43(4):869-892.

13. HARVEST, G. 2009. Biomarkers of adiponectina: Plasma protein variation and genomic DNA polymorphisms. Biomark Insights. 4(1):23-33.

14. IGLESIA DEL SOL, A.; BOTS, M.L.; GROBBEE, D.E.; HOFMAN, A.; WITTERMAN, J.C. 2002. Carotid intima-media thickness at different sites: relation to incident myocardial infarction; The Rotterdam Study. Eur Heart J. 23(6):934-940.

15. JOHNSON, B.D.; DWYER, K.M.; STANCZYK, F.Z.; BITTNER, V.; BERGA, S.L.; BRAUNSTEIN, G.D.; AZZIZ, R.; YANG, Y.C.; HALE, G.E.; BAIREY, C.N.M. 2010. The Relationship of menopausal status and rapid menopausal transition with carotid intima-media thickness progression in women: A report from the Los Angeles atherosclerosis study. J. Clin. Endocrinol. \& Metab. 95(9):4432-4440.

16. KAMPOLI, A.M.; TOUSOULIS, D.; ANTONIADES, C.; SIASOS, G.; STEFANADIS, C. 2009. Biomarkers of premature atherosclerosis. Trends Mol. Med. 15(7):323-322.

17. KOTANI, K.; CHEN, J.T.; TANIGUCHI, N. 2011.The relationship between adiponectin and blood pressure in premenopausal and postmenopausal women. Clin. Invest. Med. 34(3):E125-E130.

18. MAC ANANEY, O.; MELLOTTE, G.; MAHER, V. 2014. Comparison of semi-automated and manual measurements of carotid intima-media thickening. Biomed. Res. Int. 531389 .doi: 10.1155/2014/531389. 
19. ONATRA, W.; GARCÍA, C.; GAMBOA, C.; SÁNCHEZ, J.; CASTRO, J. 2006. Prevalencia del Síndrome Metabólico en mujeres posmenopáusicas en dos centros clínicos de Bogotá. Rev. U.D.C.A Act \& Div. Cient. 9(1):43-53.

20. OUCHI, N.; PARKER, J.L.; LUGUS, J.J.; WALSH, K. 2011. Adipokines in inflammation and metabolic disease. Nat. Rev. Immunol. 11(2):85-97.

21. RECKELHOTFF, J.F. 2004. Basic research into the mechanism responsible for postmenopausal hypertension. Int. J. Clin. Suppl. 139(1):13-19.

22. ROYER, M.; CASTELO-BRANCO, C.; BLÜMEL, J.E.; CHEDRAUI, P.A.; DANCKERS, L.; BENCOSME, A.; NAVARRO, D.; VALLEJO, S.; ESPINOZA, M.T.; GÓMEZ, G.; IZAGUIRRE, H.; AYALA, F.; MARTINO, M.; OJEDA, E.; ONATRA, W.; SAAVEDRA, J.; TSEROTAS, K.; POZZO, E.; MANRIQUEZ, V.; PRADA, M.; GRANDIA, E.; ZUNIGA, C.; LANGE, D.; SAYEGH, F.; COLLABORATIVE GROUP FOR RESEARCH OF THE CLIMACTERIC IN LATIN AMERICA. 2007. The US National Cholesterol Education Programme Adult Treatment Panel III (NCEP ATP III): prevalence of the metabolic syndrome in postmenopausal Latin American women. Climateric 10(2):164-170.

23. RUIZ, A.; MORILLO, L.E. 2004. Epidemiologia Clínica: Investigación clínica aplicada. Editorial Panamericana. BogotÁ. p.509.

24. SOULES, M.R.; SHERMAN, S.; PARROTT, E.; REBAR, R.; SANTORO, N.; UTIAN, W.; WOODS, N. 2001. Executive summary: Stages of Reproductive Aging Workshop (STRAW). Fertil. Steril. 76(5):874-878.
25. WEBER, C.; NOELS, H. 2011 Atherosclerosis: current pathogenesis and therapeutic options. Nature Medicine. 17(11):1410-1422.

26. WU, Y.; ZHENG, C.; CHEN, D.; XIE, M. 2015.Investigation of the change of adiponectin level with menopause status in middle aged women and its relationship with androgen. Zhonghua Fu Chan Ke Za Zhi. 50(5):356-360.

27. YANG, W.S.; CHUANG, L.M. 2006. Human genetics of adiponectin in the metabolic syndrome. J. Mol. Med. 84(2):112-121.

28. YAMAUCHI, T.; KAMON, J.; MINOKOSHI, Y.; ITO, Y.; WAKI, H.; UCHIDA, S.; YAMASHITA, S.; NODA, M.; KITA, S.; UEKI, K.; ETO, K.; AKANUMA, Y.; FROGUEL, P.; FOUFELLE, F.; FERRE, P.; CARLING, D.; KIMURA, S.; NAGAI, R.; KAHN, B.B.; KADOWAKI, T. 2002. Adiponectin stimulates glucose utilization and fatty-acid oxidation by activating AMP-activated protein kinase. Nat Med. 8(11):1288-1295.

29. YIANNIKOURIS, F.; GUPTE, M.; PUTNAM, K.; CASSIS, L. 2010. Adipokines and blood pressure control. Curr. Opin. Nephrol. Hypertens. 19(2):195-200.

30. ZHANG, B.C.; LIU, W.J.; CHE, W.L.; XU, Y. 2012. Serum total adiponectin level and risk of cardiovascular disease in Han Chinese populations: a meta-analysis of 17 case-control studies. Clin. Endocrinol. 77(3):370378.

Recibido: Abril 3 de 2016

Aceptado: Octubre 28 de 2016

Cómo citar:

Onatra Herrera, W.; Sánchez Angarita, J.; Parra Pineda, M.; Amaya Guio, J.; Laverde, G. 2016. Evaluación de la adiponectina y su correlación con la ìntima media carotidea en mujeres peri y post menopaùsicas. Hospital de Engativá Bogotá. Rev. U.D.C.A Act. \& Div. Cient. 19(2): 257-266. 